\title{
Quantitative Annular Dark-Field Imaging at Atomic Resolution
}

\author{
Shunsuke Yamashita ${ }^{1,2}$, Shogo Koshiya ${ }^{1}$, Kazuo Ishizuka ${ }^{1,3}$ and Koji Kimoto ${ }^{1,2}$ \\ 1. National Institute for Materials Science, 1-1 Namiki, Tsukuba, Ibaraki, Japan \\ 2. Department of Applied Chemistry, Kyushu University, 1-1 Namiki, Tsukuba, Ibaraki, Japan \\ ${ }^{3 .}$ HREM Research Inc., 14-48 Matsukazedai, Higashimatsuyama, Saitama, Japan
}

Quantitative annular dark-filed (ADF) imaging in scanning transmission electron microscopy (STEM) enables us to identify even the type and number of atoms of local crystal structures. A quantification procedure of ADF images was proposed by LeBeau and Stemmer, in which the signal at each pixel is placed on an absolute scale by normalizing the current reaching an ADF detector by the incident probe current [1]. The procedure made possible a direct comparison between experimental and simulated ADF images without any arbitrary scaling parameters. We evaluated a nonlinear response of an ADF signal detection system, which was not previously analyzed, and established a quantification procedure applicable to wider experimental conditions [2-3]. Using our procedure, we acquired quantitative ADF images of a few nanomaterials and compared with simulated images to investigate how accurately the scattering intensities match between experiments and simulations.

We used a $\operatorname{Titan}^{3}$ microscope (FEI) equipped with spherical aberration correctors (DCOR and CETCOR, CEOS) operating at an acceleration voltage of $80 \mathrm{kV}$. An ADF detector (Model 3000, Fischione) and an analog-to-digital (A/D) converter (DigiScan II, Gatan) were used. The nonlinear response of the ADF signal detection system was evaluated, and relationship between an ADF detector current $\left(I_{A D F}[\mathrm{pA}]\right)$ and an ADF image signal ( $S_{A D F}$ [count]) was measured. The incident probe current $I_{0}$ was measured in each experiment using a charge-coupled device (CCD) camera (UltraScan, Gatan), whose conversion efficiency ( 10 counts/e $\mathrm{e}^{-}$was measured in advance. Quantitative contrasts $Q_{A D F}$ [\%], i.e. $I_{A D F}$ normalized by $I_{0}$, were calculated from $S_{A D F}$ using the nonlinear response. The quantification procedure was performed using an in-house DigitalMicrograph (Gatan) scripts.

The range of ADF detection angle should be accurately measured because of its importance for quantitative comparison with simulation. The ADF inner angle was measured by scanning an incident probe on the ADF detector. Note that the ADF outer angle ( $200 \mathrm{mrad})$ is limited by the aperture in the microscope column above the ADF detector. We measured the actual outer angle by observing the shadow of the objective aperture, whose position was mechanically calibrated, as shown in Fig. 1b. In spite of the distorted diffraction pattern at higher angle (Fig. 1a), evaluated outer angle is almost round. The STEM image simulation was performed using a multislice program (xHREM with STEM Extension, HREM), in which defocus spread and residual aberrations (up to 5th order) were taken into account.

Figure 2 shows (a) a quantitative ADF image of graphene with 1-4 layers and (b) the histogram of the quantitative ADF image. The mean contrast, which was measured by averaging the value in areas including several unit cells, was $0.054 \%$ at a single-layer region. Since the mean value of a simulated image was $0.053 \%$, the mean quantitative contrast exhibited excellent agreement between experimental and simulated images.

Next we examined atomic-resolution ADF images of a single layer graphene. To reproduce precise ADF image profiles, an effective source distribution, which corresponds to a demagnified source image on the specimen, should be convoluted with a simulated image. Although a Gaussian function has been often utilized as the effective source distribution, we have found that the linear combination between Gaussian 
and Lorentzian well reproduces experimental results, as shown in Fig. 3. We also found that there is a small systematic deviation, which is probably due to time-dependent low-order aberrations. Highlystable microscope system and/or real-time aberration assessment are required for the advanced quantitative STEM imaging at atomic resolution.

\section{References:}

[1] J M LeBeau and S Stemmer, Ultramicroscopy 108 (2008), p. 1653.

[2] S Yamashita et al, Microscopy 64 (2015) 143 (doi: 10.1093/jmicro/dfu115).

[3] S Yamashita et al, Microscopy 64 (2015) 409 (doi: 10.1093/jmicro/dfv053).

[4] This study was partly supported by the JST Research Acceleration Program and the Nano Platform Program of MEXT, Japan. The authors thank Dr. Nagai, Mr. Kurashima and Ms. Ohwada for support in the STEM experiments.
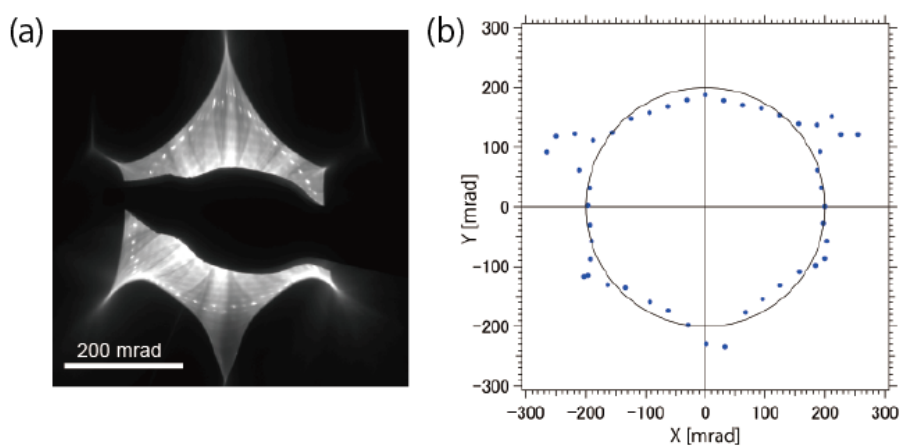

Figure 1. (a) Diffraction pattern acquired at a short camera length of $29.5 \mathrm{~mm}$. Specimen is thick $\mathrm{SrTiO}_{3}$ (001). The central area is covered by a beam stopper. The positions of outer spots are highly distorted by post specimen lenses. (b) Highest detectable angles measured using objective aperture for image-forming lens. A solid circle corresponds to 200 mrad.
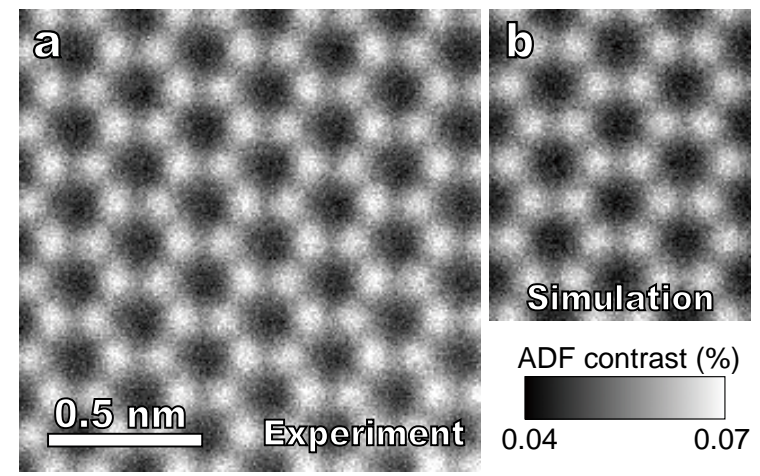

Figure 3. Quantitative ADF imaging of single-layer graphene; (a) experimental and (b) simulated images. The simulation implements defocus spread, residual aberrations (up to 5th order), shot noise and effective source distribution.
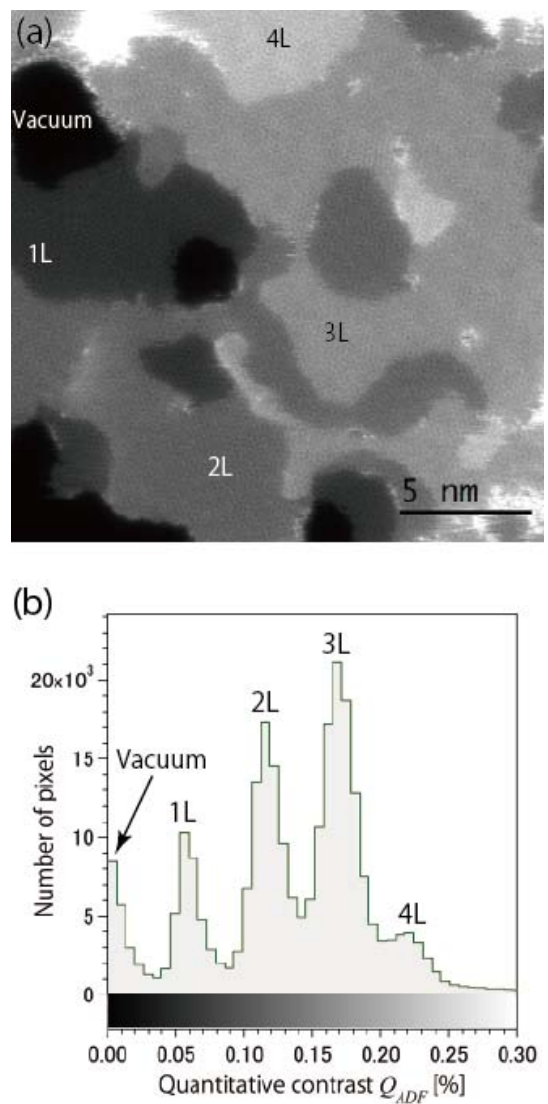

Figure 2. (a) Quantitative ADF image of multilayer graphene. The contrast scale is set in the range of $0.0-0.3 \%$. (b) Histogram of quantitative contrast, showing five discrete peaks corresponding to vacuum and 1-4 layer regions. 